\title{
Verdrängte Orte und einverleibte Bilder Die Antropofagia-Bewegung im Spiegel transregionaler Bilderwanderungen
}

\section{Un peu d'Humour?}

Als 1931 nach jahrelanger Planung die Pariser Kolonialausstellung eröffnet, ist das Echo in den Medien stark. Das parallel zur Ausstellung lancierte Journal de l'exposition coloniale ist nur eine Stimme im Gewirr der sich überschlagenden Meldungen. Eine auf der vorletzten Seite der Juni-Ausgabe platzierte Annonce hätte kaum mehr Gewicht gehabt, trüge sie nicht den sensationellen Titel Exposition de Blancs chez les Noirs. Der Artikel stammt von Max Deauville und berichtet von vorangeschrittenen und nunmehr abgesegneten Plänen, die »Einheimischen" aus der Metropole 1934 unter den Palmen von Timbuktu auszustellen. ${ }^{1}$ Unter der Leitung von André Citroën würde ein Konvoi von Autofahrzeugen die »weißen Urbewohner» in die Ferne transportieren. Vor Ort seien neben einem "Stand Peugeot" auch Konzerte sowie Spektakel mit diversen Schaustellerinnen aus Paris vorgesehen. Trotz aller Euphorie und Präzision, mit der die Neuigkeiten angekündigt werden, kann für den Leser kein Zweifel bestehen: Der Artikel ist Satire; es geht, wie ein Hinweis in der rechten oberen Ecke bestätigt, um »un peu d'Humour...«. Das auf die Spitze getriebene Détournement hätte alle Ingredienzen, produktive Verwirrung zu stiften, wären da nicht die sechs begleitenden Darstellungen (Abb.1). In ihnen scheinen alle schwarzen Akteure klischeehaft verzerrt und auf Marionetten verdutzter Zuschauer reduziert, wohingegen die weißen Präsentatoren als belehrende Wissensübermittler hervortreten. Das letzte Bild zeigt die "Rache des Weißen Mannes«: eine umgekehrte Kannibalenszene, aus der der Europäer zwar nackt, aber mit dem Leben davonkommt; der Andere, der Fremde, ist und bleibt Opfer. Trotz demonstrativer Umkehrung scheinen die Hierarchien bewahrt. Die Bilder sind ungewohnt und dennoch vertraut.
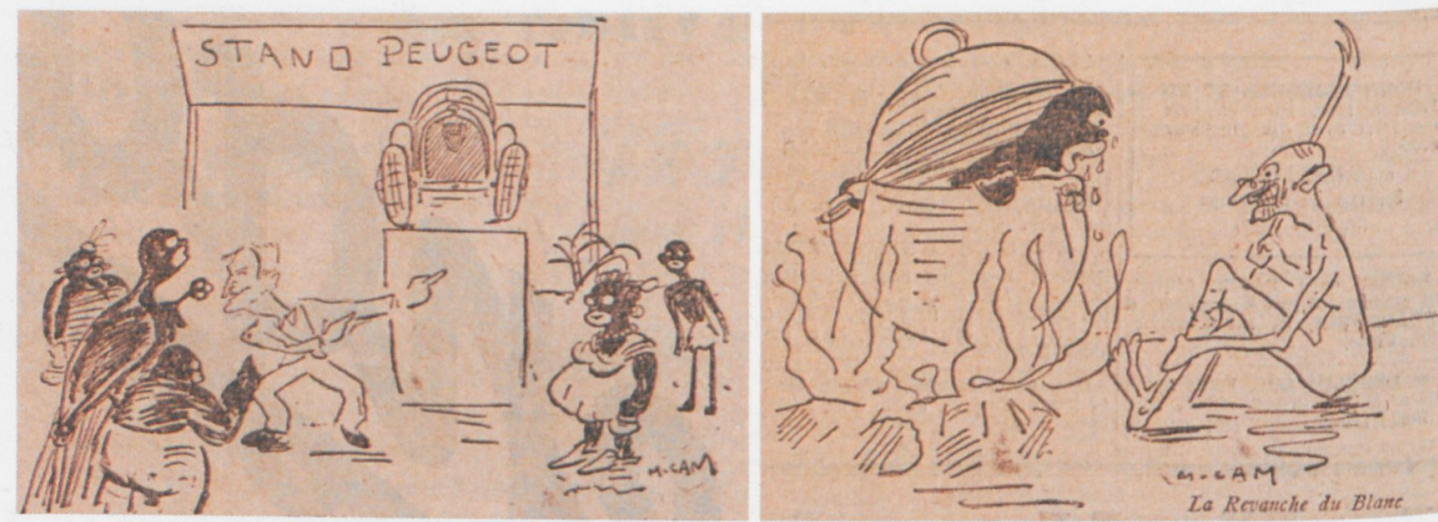

1 Zwei Darstellungen aus dem Journal de l'Exposition coloniale, Juni 1931 
Die Zeichnungen erweisen sich als Karikatur eines unmöglichen Détournements, in dem die tradierten Macht- und Rollenverhältnisse der Kolonialherrschaft umso vehementer durchscheinen. Sie sind ein negatives Pendant zu den von Viktoria Schmidt-Linsenhoff im ersten Kapitel ihres Buches Ästhetik der Differenz analysierten »Künstler- und Entdeckerblicken«, die inmitten der europäischen Kolonialkulturen ästhetische Spielräume für Selbstreflexion und Subversion eröffnen. ${ }^{2}$ Gerade weil sie vorgeben, Differenz anders erfahrbar zu machen, lassen die französischen Karikaturen kaum mehr »difference within $\star^{3} \mathrm{zu}$ und bestärken stattdessen hegemoniale Diskursmuster im Medium des Bildes. Sie antworten auf höchst defensive Art und Weise auf die beeindruckende Bandbreite interkultureller Begegnungen, die in den sogenannten Années Folles in Paris zusammenkam - von forcierten und pervertierten Beziehungen im Rahmen der zzoo humains bis hin zu idealisierten, imaginierten Bewegungen und realen Kontakten im Umkreis der Avantgarde-Künstler. Wie das Beispiel Tarsila do Amarals aus São Paulo zeigt, war die Ambivalenz der Begegnung auch für die Erfahrung der aus anderen Ländern angereisten Künstler und Intellektuellen prägend. Ihre Bilder aber zeugen von einer anderen Art der Auseinandersetzung mit Vorbildern und Vorurteilen; sie zeugen von einer anderen Art des Umgangs mit der Erinnerung an das nie Gesehene.

\section{A Negra - Tarsila do Amaral in Paris}

Gemeinsam mit der Malerin Anita Malfatti und den Schriftstellern Menotti del Picchia, Mário de Andrade und ihrem Ehemann Oswald de Andrade zählt Tarsila do Amaral zu dem sogenannten Grupo dos Cinco, der für die Begründung des Modernismo in Brasilien bekannt ist. In Amerika gab es die Armory Show (New York, 1913), in Brasilien die Semana da Arte Moderna (São Paulo, 1922); letztere fand im Kontext

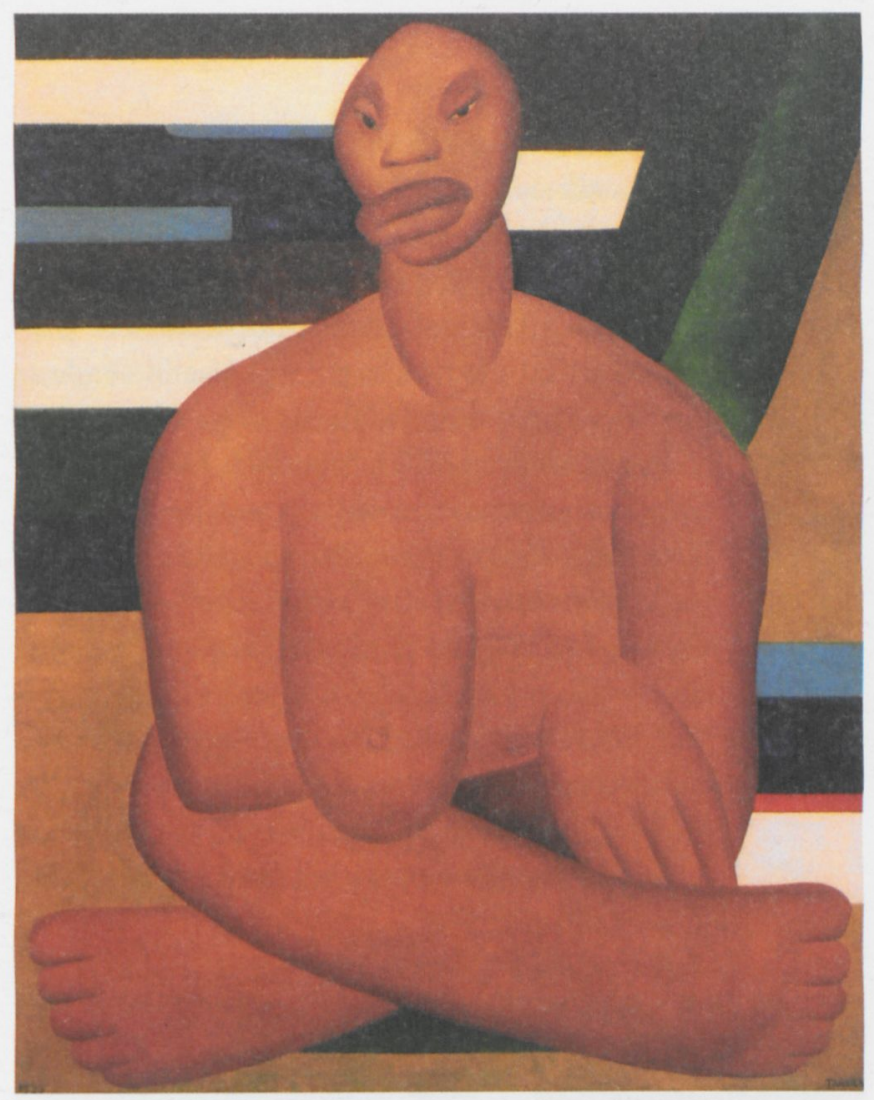

2 Tarsila do Amaral, A Negra, 1923, Öl auf Leinwand, $100 \times 81,3 \mathrm{~cm}$. São Paulo, Museu de Arte Contemporânea da Universidade de Săo Paulo

der Hundertjahrfeier der brasilianischen Unabhängigkeit statt und gilt als Initialzündung der Moderne. ${ }^{4}$ Im Jahr darauf schuf Tarsila do Amaral A Negra (Abb. 2): die Darstellung einer schablonenhaft reduzierten schwarzen Figur mit fülliger Brust und dicken Lippen. Sie ist im Schneidersitz dargestellt, beide Hände vor dem Bauch gekreuzt, als hielten sie eine Brust und verdeckten die andere; ihr Blick scheint direkt an den Betrachter gerichtet. Die schwarze Frau ist in erdigen Tönen dargestellt, während im Hintergrund mehrfarbige Linien ein stark schematisiertes Palmenblatt abstrakt zu umranden scheinen.

Tarsila do Amaral hatte sich 1921 in der Académie Julian eingeschrieben, zur Zeit von $A$ Negra war sie Schülerin von Fernand Léger. In einem vielzitierten Brief berichtet die Künst- 
lerin ihrer Familie von den Eindrücken aus Paris, wo sie neben Schlüsselfiguren der europäischen Avantgarde auch Akteure des New Negro Movement getroffen haben dürfte: "Ich fühle mich jedes Mal mehr brasilianisch: [....] Denkt nicht, dass diese brasilianische Tendenz in der Kunst hier schlecht angesehen wird. Ganz im Gegenteil. Was man sich hier wünscht, ist, dass jeder seinen Beitrag aus dem eigenen Land mitbringt. So erklärt sich der Erfolg der russischen Ballette, der japanischen Stiche, der Musik der Schwarzen. Paris hat genug von der Pariser Kunst. ${ }^{5} \mathrm{Zu}$ einem Zeitpunkt, als namhafte Vertreter der brasilianischen Intelligentsia den Fortschritt des Landes mittels rassistischer Whitening-Theorien über eine zunehmende `Mulattisierung` der Gesellschaft zu beschwö-

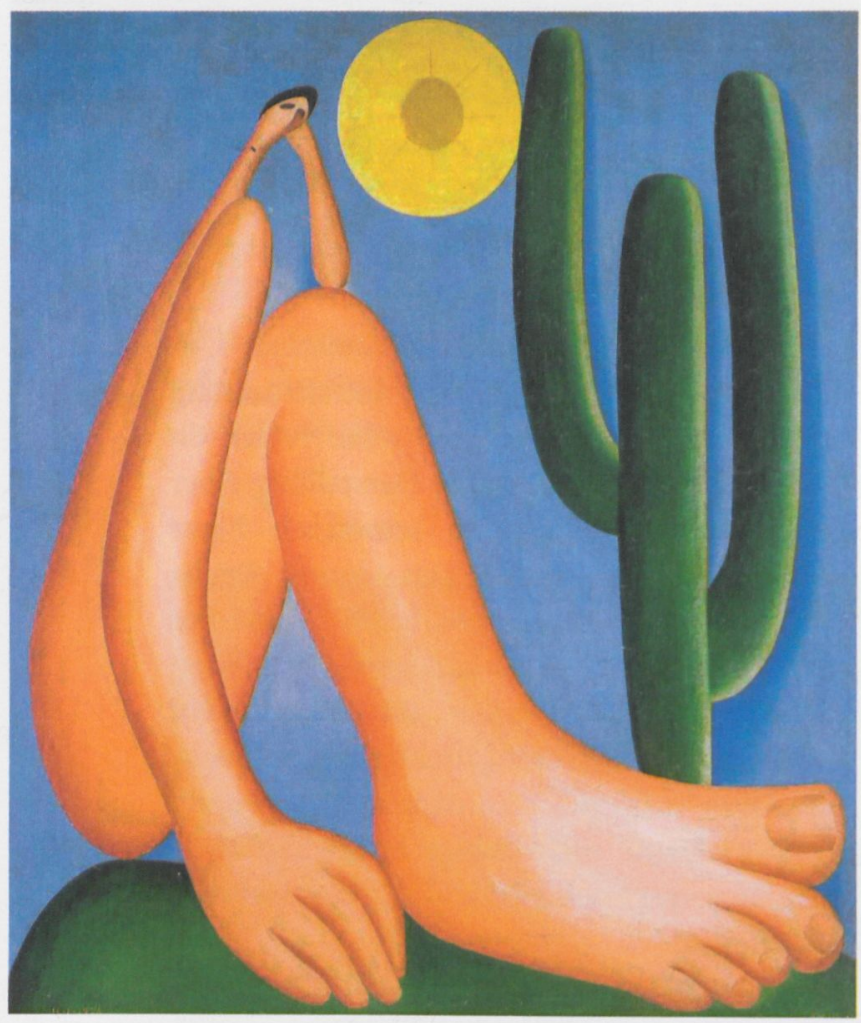

3 Tarsila do Amaral, Abaporu, 1928, Öl auf Leinwand, $85 \times 72 \mathrm{~cm}$. Buenos Aires, Museo de Arte Latinoamericano de Buenos Aires, Fundación Constantini ren suchten (und damit die Strukturen eines internalisierten Kolonialismus bestärkten), hebt A Negra die schwarze Frau in ihrer prallen Körperlichkeit als bildwürdiges Thema hervor, ohne sie zu folklorisieren.

Der brasilianische Modernismo ist von Anfang an mit dem beschäftigt, was viele Jahre später von Kobena Mercer, Stuart Hall u. a. als »burden of representation ${ }^{6}$ kritisch zur Diskussion gestellt wird. Die Frage der sbrasilidade wurde zunächst im Zusammenhang der Literatursprache aufgeworfen und fand in der Phase Pau-Brasil auf starke Weise auch bildhaft ihren Niederschlag. Sie impliziert eine grundlegende Auseinandersetzung mit der eigenen Identität und der Relevanz europäischer Einflüsse, die sich angesichts der langen Kolonialvergangenheit jedoch äußerst komplex gestaltete. Die neuen künstlerischen Tendenzen aus Europa boten zwar einen Ausweg aus Akademismus und Folklore, stellten aber kulturelle, politische und historische Konfliktlinien zur Diskussion. A Negra zeigt bereits an, wie im Medium der Kunst Wege zur produktiven Durchdringung dieser ambivalenten Lage erschlossen wurden.

\section{III. »Tupy, or not tupy«}

Zwei Jahre nach A Negra entsteht Abaporu (Abb. 3), ein Gemälde, das heute zu den Ikonen der lateinamerikanischen Kunst zählt: »eine einzelne, monströse Figur, immense Füße, auf einer grünen Ebene sitzend; der gebeugte Arm auf dem Knie ruhend, die Hand das Federgewicht des winzigen Kopfes tragend. Im Vordergrund, ein in eine absurde Blume zersprengender Kaktus«? In ihrer pointierten Beschreibung verzichtet Tarsila do Amaral auf allegorische Projektionen, um den programmatischen Bruch mit der akademischen Tradition der brasilianischen Eliten zu betonen. Der Titel Abaporu bedeutet in der Tupí-Sprache »Mann, der Menschen isst«. Es sollte die Initialzündung zur Antropofagia- 
Bewegung werden. Als Geschenk an ihren Ehemann Oswald de Andrade weitergegeben, taucht das Bild kurz darauf als Umrisszeichnung wieder auf, als dieser im Mai 1928 in der ersten Ausgabe der Revista de Antropofagia, die auf ihrem Titelbild eine Illustration zu Hans von Stadens berühmtem Reisebericht trägt, das Anthropophagische Manifest veröffentlicht. ${ }^{8}$

Verschiedentlich haben Künstler der Avantgarde in Europa auf die Rede vom Kannibalismus rekurriert, um sich in humoristischer, provokatorischer oder metaphorischer Geste für »das große Außerhalb, die große Andersheit ${ }^{9}$ der modernen Zivilisation auszusprechen (Francis Picabia, Tristan Tzara, Filippo T. Marinetti, Blaise Cendrars, Marcel Duchamp, Man Ray, Alfred Jarry etc.). Im Kontext der brasilianischen Avantgarde erfährt die »klassische Figur der Transgression ${ }^{10}{ }^{10}$ jedoch eine besondere Prägung; sie ist historisch und kulturell verankert, konkreter und philosophischer zugleich. Die Grundidee des Manifestes basiert auf einer Inversion: Das europäische Zerrbild des primitiven Kannibalen, »die kolonialistische Metapher par excellence ${ }^{11}$, wird zum Signum des Widerstandskämpfers erklärt, um der sowohl historisch als auch geographisch vernachlässigten Peripherie eine neue Aktualität abzugewinnen. Das Programm verspricht Emanzipation durch Absorption, Verflechtung und Transformation; es erfasst alle Bereiche der Kultur und ist auch heute noch von großem Einfluss.

Die Bewegung richtet sich gegen die Übermacht der kolonialistischen Einwirkungen, fordert jedoch im Gegensatz zu zeitgleichen nationalistischen, xenophobischen Strömungen (wie die einwanderfeindliche Gegenbewegung Verdamarelo oder die um das Symbol des pflanzenfressenden Tapirs als Totemtier Brasiliens konzipierte AntaGruppe $^{12}$ ) keine radikale Ablehnung der europäischen Einflüsse - sie unterstreicht vielmehr "die Notwendigkeit, das Nationale in dialogischdialektischem Zusammenhang mit dem Universalen zu denken $\aleph^{13}$. Das Manifest propagiert dafür das Prinzip der Einverleibung als kulturelle Strategie: Nach dem Vorbild der brasilianischen Tupinambá-Indianer, die Teile ihrer wichtigsten Feinde in Stammesritualen aufaßen, um sich deren Mut, Kraft und Stärke anzueignen, gelte es, ausgewählte Elemente der fremden Kultur einem Totem gleich in sich aufzunehmen (statt sie von vorne herein abzuweisen oder bloß zu kopieren) und sie mit den indigenen und afrikanischen Überlieferungen zu vermengen. Es geht hier also nicht um Einfluss oder Transfer, sondern um realen Kontakt, um Austausch und Begegnung. Die Rede von der Anthropophagie ist abstrakt, speist sich aber aus der Unhintergehbarkeit körperlicher Erfahrungen und betont die Bedingtheit individueller Identitäten. Sie verwehrt sich gegen »die Illusion des Sich-Selbst-Genügen[s] oder der Autonomie « und beharrt auf das "Immer-schonverwiesen-sein auf den Anderen im Verhältnis zu ihm ${ }^{14}{ }^{14}$ Anthropophagie heißt Verschlingen, Verzehren und Zerstören, aber auch Métissage, Vermischung und Dégustation: "L'anthropophagie est le désir de l'autre en tant qu'autre, dans la voie de l'interdépendance, de la relation. Ouverture dévoratrice, mais dévoration critique, car soumise à sélection: ne pas manger n'importe quoi! « ${ }^{15}$

Das Manifest ist mit viel Humor geschrieben und vereint eine Fülle (kultur-)historischer und intellektueller Referenzen, die mit subtilem Wortwitz und kreativ-assoziativ zueinander in Beziehung gesetzt werden. Genährt durch die Lektüre von Sigmund Freud, Jean-Jacques Rousseau, Michel de Montaigne, Lucien LévyBruhl und anderen wird die lange Zeit unterdrückte indigene Kultur samt ihrer von außen an sie herangetragenen Konnotationen als Kulturell-Unbewusstes in Erinnerung gerufen und zur symbolischen Identifikationsfolie erhoben. Damit markiert das Manifest eine doppelte Bewegung: die Umwandlung europäischer Zerrbilder und die Abwendung von verklärenden Tendenzen des brasilianischen Indianismus. Es galt, ein Gegenmodell zur passiven Assimilation zu entwerfen, ohne einen neuen Essenzialismus 


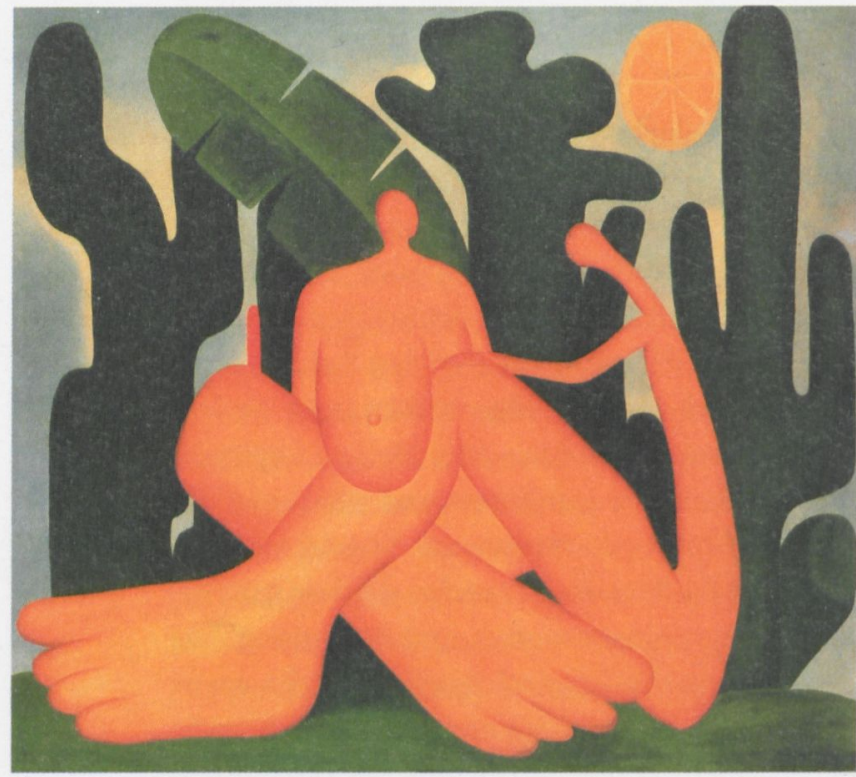

4 Tarsila do Amaral, Antropofagia, 1929, Öl auf Leinwand, $126 \times 142 \mathrm{~cm}$. São Paulo, Fundação José e Paulina Nemirovsky

$\mathrm{zu}$ lancieren. Die verschlingende Metamorphose ist gleichermaßen kreative Praxis und politisches Konzept - auch für das Manifest selbst, das die doppelte Prämisse untermauert, indem es das Prinzip der Einverleibung thematisch als Phänomen und erzähltechnisch als Strategie aufeinander bezieht: "Tupy, or not tupy that is the question ${ }^{16}{ }^{16}$ lautet einer der Schlüsselsätze der projizierten Einverleibung, die auf diesem Wege sogleich performativ vollzogen wird. Das emblematische Wortspiel ist Programm.

\section{Andere Moderne?}

Der brasilianische Modernismo ist die Bewegung einer privilegierten, kultivierten Klasse mit ausgezeichneten Sprachkenntnissen und Zugang zu fremdsprachiger Literatur. Seine Akteure verkehrten zwischen Europa und Lateinamerika und agierten ihrerseits als wichtige Kulturmittler einer frühen transkulturellen Moderne. Tarsila do Amaral übernahm wiederholt Kaufaufträge für ihre Freunde aus Brasilien, sammelte selbst bedeutende Werke von Fernand
Léger, Constantin Brâncusi, Giorgio de Chirico, Robert Delaunay und anderen und unterstützte den interkulturellen Austausch über Einladungen namhafter Intellektueller nach Brasilien. Sie stand in enger Beziehung zu einer Großzahl der damals tonangebenden Akteure aus Paris, über deren Aktivitäten sie regelmäßig für die brasilianische Presse berichtete. ${ }^{17} 1928$ fand bereits ihre dritte Einzelausstellung in Paris statt (die erste erfolgte 1926, in Brasilien hingegen erst 1929). Ein weiteres Schlüsselbild entstand: Antropofagia (Abb. 4), eine Art Selbstkannibalisierung von A Negra und Abaporu.

Das Gemälde unterstreicht den konstitutiven Doppelcharakter der Bewegung: In der Forderung nach einem selbstbewussten Bezug auf die eigene Tradition wird das Begehren nach dem fremden Anderen nicht unterdrückt, sondern bestärkt. Die Antropofagia-Bewegung ist zukunftsorientiert, propagiert aber keinen naiven Fortschrittsoptimismus. Anders als der Futurismus, den Amaral selbst einmal als »Aufschrei der Revolte gegen die Vergangenheit« anprangerte, fordert die Antropofagia-Bewegung keine "Tabula Rasa zur Konstruktion einer neuen Welt für einen neuen Menschen «. $^{18}$ Statt aus einer Geste der Selbstverneinung speist sich die Bewegung aus der Wertschätzung der eigenen Kultur- und Naturgeschichte. Auch jenseits von Primitivismus und Surrealismus boten sich ihr in Europa dafür fruchtbare Anregungen. Vor dem Hintergrund einflussreicher Kolonialausstellungen und deren kritischer Rezeption war eine komplexe Konstellation gegeben, in der Konzepte von Zentrum und Peripherie einem permanenten Aushandlungsprozess ausgeliefert waren. Im Rahmen der Erfahrungen einer transkulturellen Moderne werden die Konturen dessen greifbar, was Édouard Glissant mit Blick auf "Mikro- und Makroklimate einer gegenseitigen Durchdringung in Sprache und Kultur« als "Chaos-Welt« umschrieb: das unerwartete Aufeinanderprallen von verschiedenen Elementen, aus dem sowohl komplexe Kulturen (»cultures 
composites«) als auch damit verbundene Ängste und Abwehrmechanismen hervorgehen. ${ }^{19}$

Die Antropofagia-Bewegung schafft Durcheinander im Projekt der Moderne. Sie beharrt auf der Idee einer dynamischen Verflechtung und entzieht sich dem Mythos stabiler Identitäten. Das Motiv der Einverleibung setzt eine Reihe fundamentaler Binome in Frage (modern/ primitiv, Original/Kopie, Zivilisation/Barbarei, Eigene/Fremde, Natur/Kultur, Vergangenheit/Gegenwart etc.): "Einerseits erscheint der andere als Anderer, andererseits lässt er sich nur im Einen als Anderer beschreiben - und wird somit Teil des Einen. $\aleph^{20}$ Mit diesem »Jenseits-der-Grenze-Phänomen« werden wichtige Ordnungsprämissen, Bezeichnungsstrategien und Kategorisierungsverfahren der Moderne in Frage gestellt: »Kannibalismus ist in der Tat ambivalent; er schafft absolute Differenz, in der Opposition von Esser und Gegessenem und gleichzeitig löst er diese Differenz durch den Akt der Einverleibung auf. ${ }^{21}$ Die AntropofagiaBewegung begrüßt eben jene Vermischung, die laut Bruno Latour konträr zum Programm der Moderne steht. Letztere versuche hybride Konstellationen zu verleugnen, führe damit aber erst recht zu deren Vermehrung; die gegenläufigen Tendenzen von Reinigung und Ent-Differenzierung sind aufs Engste aufeinander bezogen: "plus on s'interdit de penser les hybrides, plus leur croisement devient possible ${ }^{22}$ Die Antropofagia-Bewegung agiert nicht im Sinne der ‘Großen Trennung, sie stiftet Konfusion inmitten des modernen Projektes der rationalisierenden `Entmischung ‘. Sie vermengt wildes Denken und modernes Denken und lässt die Konturen einer "peripheren Moderne ${ }^{23}$ erkennbar werden. Eingedenk der Notwendigkeit, neue Präsentismen abzuwehren, bieten sich hier interessante Anknüpfungspunkte $\mathrm{zu}$ jüngeren Diskussionen um Kontaktzonen, Hybridität, Migration oder Transkulturalismus. Auch für die Kunstgeschichte gehen damit wichtige Perspektivwechsel einher. ${ }^{24}$ Bildtheoretische Per- spektiven eröffnen sich nicht zuletzt mit Blick auf die Arbeiten Aby Warburgs; auch er, so Georges Didi-Huberman, »desorientiert die Geschichte, [...] öffnet und kompliziert sie. स $^{25}$

\section{Bildwandlungen und Bildwanderungen}

Das unvollendet gebliebene Mnemosyne-Projekt, mit dem die »Einverseelung vorgeprägter Ausdruckswerte [...] von der hilflosen Versunkenheit bis zum mörderischen Menschenfraß ${ }^{26}$ anschaulich werden sollte, korrespondiert nicht allein zeitlich mit der Antropofagia-Bewegung. Im Bemühen einer transhistorischen Verflechtung zwischen Phantasie und Vernunft, Mythos und Logos, und nicht zuletzt in den Bezeichnungsmodalitäten, die Warburg dafür wählte, werden zwar aufschlussreiche Berührungen manifest - aber auch bedeutende $\mathrm{Ab}$ weichungen, die sich den jeweils unterschiedlichen Schauplätzen verdanken, je nachdem ob sie tendenziell eher einer "westlichen Moderne» oder einer "transkulturellen globalen Moderne" zugehören. ${ }^{27}$ Warburgs Aufsatz zu Édouard Manets Frühstück im Grünen (Abb. 5), mit dem das Mnemosyne-Projekt abschließen sollte, ist in diesem Zusammenhang besonders interessant. Der Text handelt von »Wandlungen und Verpuppungen $\aleph^{28}$ der europäischen Kulturgeschichte und wurde wenige Monate nach $\mathrm{Aba-}$ poru verfasst. Mit Manets Frühstück im Grünen wählte Warburg »ein zentrales Schwellenwerk der Moderne ${ }^{29}$, das als radikaler Bruch mit der Vergangenheit gefeiert wurde: als »Paradigma avantgardistischer, das heißt mit den tradierten Konventionen bewusst brechender und folglich vom Publikum abgelehnter Malerei« «. ${ }^{30}$ Für Warburg entspringt die Neuartigkeit Manets jedoch keiner voraussetzungslosen Wendeerscheinung, sie ist vielmehr Resultat folgenreicher Bildwandlungen und Bildwanderungen. Wie er aus der Zusammenschau mit einem Kupferstich der Renaissance und einem antiken Sarkophag 


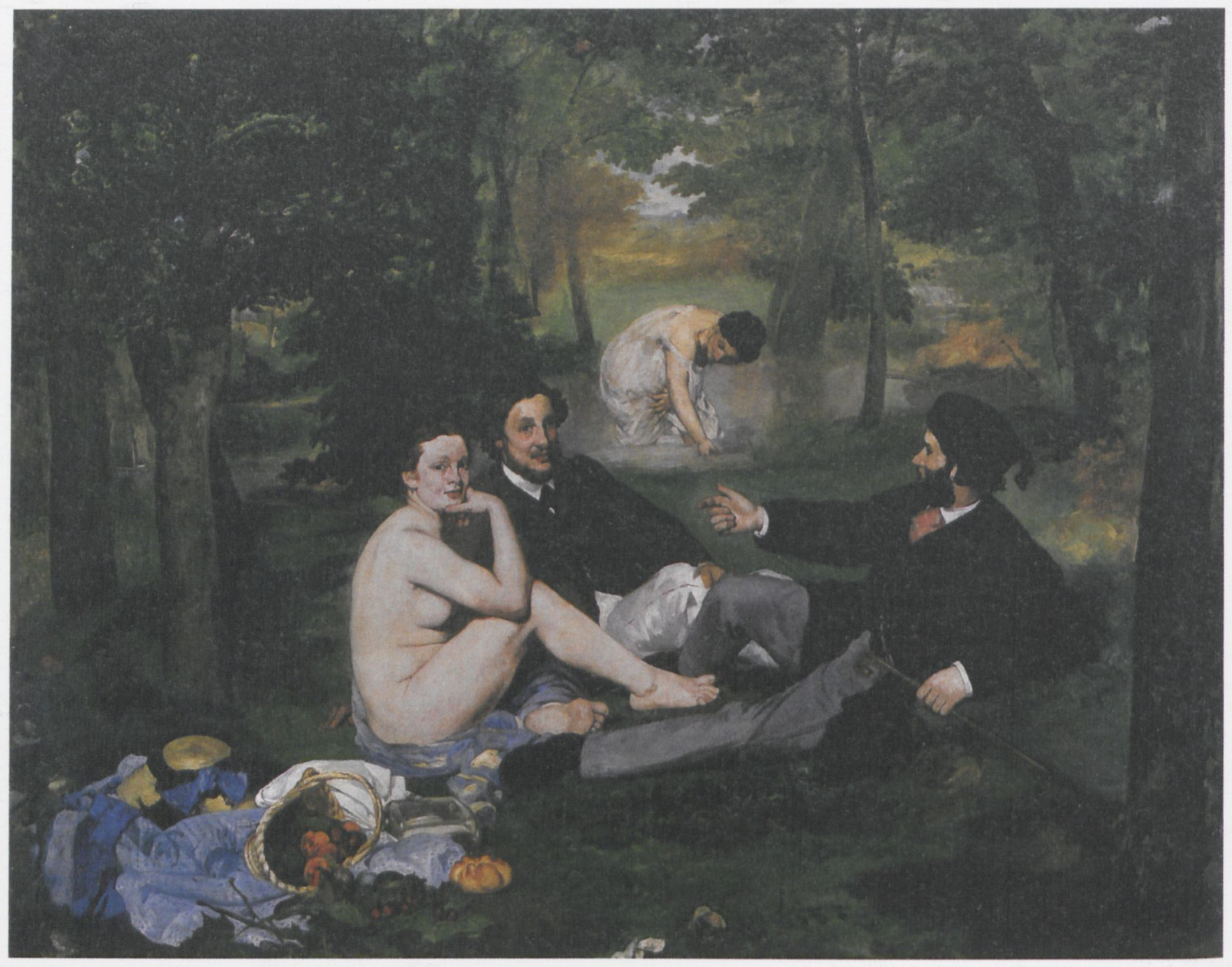

5 Édouard Manet, Le Déjeuner sur l'herbe, 1863, Öl auf Leinwand, $208 \times 264,5$ cm. Paris, Musée d’Orsay

ableitet, lässt sich mit Manets Gemälde belegen, »dass Teilhabe am geistigen Gesamterbgut erst die Möglichkeit schafft, einen neue Ausdruckswerte schaffenden Stil zu finden, weil diese ihre Durchschlagskraft nicht aus der Beseitigung alter Formen, sondern aus der Nuance ihrer Umgestaltung schöpfen ${ }^{31}{ }^{31}$

Warburg erörtert das »Problem der überlebenden Kraft antikisierender Vorstellungen $\aleph^{32}$, indem er ältere Vorbilder hinzuzieht, darunter auch den Kupferstich Das Urteil des Paris von Marcantonio Raimondi, der auf einen verlorenen Karton Raffaels zurückgeht, dem seinerseits das Relief eines Sarkophags aus der Villa Medici in Rom zugrunde lag. In der $\mathrm{Zu}$ sammenschau von antiker Bildwelt und italie- nischem Mittler werden angesichts von Manets Nachbild aufschlussreiche Verrückungen sichtbar, namentlich in der Transformation einer Figurengruppe von drei lagernden Nymphen: Die »minutiöse Vergleichung der Allüren im Halbgötterpublikum « ${ }^{33}$ lasse erkennbar werden, inwiefern die Überlieferung das pagane Bildmaterial umformt. Der vergleichende Blick dient jedoch nicht der Bescheinigung einer bloßen Motivgeschichte oder dem Nachweis von Form-Analogien. Vielmehr geht es darum, hierarchische Übertragungsmodelle einer reinen Einflussgeschichte zu überwinden und jenseits strikter Kausalzusammenhänge tiefer gehende Bildbeziehungen in den Blick zu rücken. Die Frage, was »formâle und sachliche Zusammen- 
hänge mit der Tradition ${ }^{34}$ aussagen, birgt bei Warburg stets auch psychologische, ethische und spirituelle Komponenten.

Anhand von wanscheinend ganz unbedeutenden Abweichungen « in subtilen Details zeigt der Hamburger Bildhistoriker, wie die Ehrfurcht vor den himmlischen Göttern schwindet. Für Warburg offenbart sich die »energetische Umverseelung " vor allem im Blickwechsel zwischen den olympischen Gottheiten und den erdgebundenen Halbgöttern: Während die antike Figurengruppe eine "schauende Ergriffenheit" bezeuge und ihren Blick hilflos gen Himmel richtet, wenden sich die Lagernden bei Raimondi und Manet der »beschauenden Aussenwelt « $\mathrm{zu} .{ }^{35}$ Dieses "Zuschauerbewußtsein" sei Ausdruck einer energetischen Inversion ${ }^{36}$ : $»$ Aus der kultisch zweckgebundenen Geste untergeordneter blitzfürchtiger Naturdämonen auf dem antiken Relief vollzieht sich über den italienischen Stich die Prägung freien Menschentums, das sich im Licht selbstsicher empfindet. ( $^{37}$

\section{Nachleben und Wiederkehr}

Manet, "der Vorwärtsschreitende«, so Warburg, gelangt zu Neuerung und Originalität »durch Rückwärtswendung «. ${ }^{38}$ Die Übernahme vererbter Formenelemente findet hier als Akt einer kreativen Integration Würdigung: Sie ist keine passive Anpassung, sondern aktive Transformation in Reaktion auf "die vorprägende Funktion heidnischer Elementargottheiten für die Entwicklung modernen Naturgefühls « ${ }^{39}$ Cornelia Zumbusch hält fest: "Manet erinnert die antiken Vorbilder in einem neuen Bild und löst sich von der Vorlage, gerade indem er sie aufnimmt $«{ }^{40}$ Analog dazu folgert Andrea Pinotti: "Emprunter signifie dès lors reprendre, non pour imiter, répéter, confirmer, mais plutôt pour transformer, varier, et, à la limite inverser. Continuer pour rompre ou rompre pour continuer? « ${ }^{41}$ Der Fokus zielt weniger auf Kontinuität und Nachahmung als auf Transformation, Auseinandersetzung und Inversion ( $>$ Umverseelung`). Der Erinnerung liegt hier eine verändernde Kraft zugrunde, die ihr Potential aus der 'Nuance ihrer Umgestaltung schöpft: »In der mnemischen Funktion trifft das Wunder der Konstanz mit dem ebenso großen Wunder der Wandlung zusammen «. ${ }^{42}$

Indem Warburg Phänomene der Wiederkehr und Fragen des Nachlebens der Bilder erörtert, drängt er tradierte Denkmuster von Einfluss und Rezeption in die Enge. Seine Erinnerungstheorie steht konträr zu den ideologischen Implikationen von kunsthistorischer Strukturanalyse und Autonomieästhetik. Das Bewusstsein für die Wanderwege der Bilder sprengt den Fokus auf das isolierte Kunstwerk. Es geht nicht, wie beispielsweise für Dagobert Frey, um dessen »Einzigartigkeit, Einmaligkeit und Erstmaligkeit«, um »Ausschließlichkeit« oder »Nichtdagewesenes $«,{ }^{43}$ sondern um Nachleben und Wiederkehr. Für Warburg sind Kunstproduktion und Kunstrezeption eng miteinander verwoben, das Vorbild wird im Nachbild aktiv und vice versa: »le proto-type comme pré-type, comme préimage, se présente aussi comme une post-image, instituée a posteriori par l'artiste moderne. « ${ }^{44}$ Vergangenheit, Gegenwart und Zukunft sind unlösbar miteinander verflochten: Erinnerung ist ‘Einverseelung`, die Rückwärtswendung eine Vergegenwärtigung, in der das Vergangene transformiert wird. Warburg widersetzt sich einem linearen Geschichtsverständnis, um anachronistische Bildgeschichten zu aktivieren. Edgar Wind kommentiert: "Die Geschichte srollt nicht einfach sab<, sondern sie vollzieht sich in Krisen, und ihre entscheidenden Ereignisse sind die >Pausen der Besinnung ${ }^{\prime}$, denen das Wagnis der Handlung folgt. Gerade in diesen Krisen aber muß sich die gestaltende Macht der Erinnerung bewähren, die durch Ergreifung und Belebung überkommener Symbole zur Besinnung aufruft oder zur Handlung treibt und so die Umkehr periodisch auslöst« ${ }^{45}$ 


\section{Erinnerungsgemeinschaft}

Jenseits formaler Berührungen zwischen Manets Frühstück im Grünen und Tarsila do Amarals Gemälden Abaporu und Antropofagia, die sich auch mit Blick auf Fragen »lastender Noch-Körperlichkeit « ${ }^{46}$ durchdenken ließen, zeigen Warburgs Mnemosyne-Projekt und die brasilianische Antropofagia-Bewegung tiefer gehende Verwandtschaftsbeziehungen. Sie begegnen sich insbesondere in ihrer Konzeption der Erinnerung als einem Akt der bewusstmachenden Vergegenwärtigung vergessener, verschütteter Potenziale. Warburgs Konzept der `Einverseelung` und Andrades Idee der Einverleibung stehen gleichermaßen im Dienste der Selbstaufklärung, »des Selbsterziehungsversuchs « ${ }^{47}$. Hier wie dort werden Metaphern für Transfer- und Aneignungsprozesse bemüht, um Möglichkeiten einer Verflechtung zu erproben, in der Affektion nicht zur Unterwerfung führt. Warburg und Andrade skizzieren das Konzept einer »Erinnerungsgemeinschaft«, die aus dem nuancierten Rückgriff auf die Vergangenheit "retrospektive Besonnenheit ${ }^{48}$ und Kraft zur Veränderung schöpft. Auch hier gilt, was Walter Benjamin mit seiner paradoxen Wendung der Bilder, die wir nie sahen, ehe wir uns ihrer erinnerten $\ll,{ }^{49}$ prägnant auf den Punkt brachte: Erinnerungstheorie und Bildverständnis stehen in engster Beziehung zueinander.

Wie die Lektüre Didi-Hubermans ihrerseits suggeriert, berühren sich die AntropofagiaBewegung und Warburgs Interesse für eine Kunstgeschichte, in der auch anthropologische Fragen des Aberglaubens Platz haben, in ihrer Desorientierung der Geschichte: »Jede Periode ist ihr eigener Knoten aus Altertümern, Anachronismen, Gegenwärtigem und Ansätzen zu

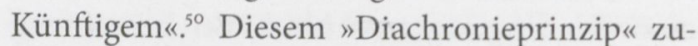
folge ist »jedes Bild eine Kreuzung aus aktuellem Anlass und Reaktion auf Vor-Bilder «. ${ }^{51}$ Mit Blick auf einen bemerkenswerten Aufsatz, den der spanische Kunsthistoriker Ángel Gonzá- lez García dem Kaktus in der bildenden Kunst widmete, ließe sich das in den Gemälden von Tarsila do Amaral wiederkehrende Pflanzenmotiv als feines Indiz für diese doppelte Verfasstheit betrachten: ein Sujet, das archaisch erscheint und zugleich ausgesprochen modern ist $\mathrm{t}^{52}$ - keine Pathosformel, aber doch ein Phantom, das wiederkehrt: „Gerade Dinge, die seit langem tot sind, spuken besonders wirkungsvoll - und auf besonders gefährliche Weise - in unserem Gedächtnis. «33 Die Wiederkehr des Verdrängten ist zentrales Movens der Anthropophagischen Initiative, die sich auf diesem Wege dezidiert zu einer mémoire involontaire bekennt.

Der Kannibale ist eines dieser Gespenster, die die Antropofagia-Bewegung in der Tradition der Avantgarde-Bewegungen humoristisch überspitzt wiederzubeleben vorgibt. Er steht pars pro toto für ein Kulturell-Unbewusstes, das im Zeichen der Kolonialisierung verdrängt, deformiert und umgedeutet wurde. Wie die Pueblo-Indianer in Warburgs Interpretation des Schlangenrituals, wenn auch auf andere Art und Weise, impliziert der Anthropophage in der Lektüre Andrades einen »Misch- und Übergangszustand», der konträr zum Latourschen Projekt der Moderne steht, indem er das "Nebeneinander von logischer Zivilisation und fantastisch magischer Verursachung« verkörpert: »in der Mitte zwischen Magie und Logos [...], zwischen Greifmenschen und Denkmenschen «. ${ }^{54}$ Auch das Manifest zelebriert ihre Vermischung. Aus jeweils anderer Perspektive, und dennoch verwandt, argumentieren Warburg und Andrade mithilfe des primitiven Menschen gegen Engpässe eines abendländischen Fortschrittsglaubens. Hier wie dort steht die Erinnerung im Zeichen der Emanzipation, die Wiederkehr im Bild ist eine kulturelle Bewältigungsstrategie. Nirgends aber geht es um ein Zurück zu verlorenen, vermeintlich unverfälschten Wurzeln, um Beginn oder Anfangspunkt: »Der Ursprung ist [...] selbst schon eine unreine Zeitlichkeit aus Mischformen und Ablagerungen, Vorauswei- 
sendem und Zerfallenem «.55 Die Geschichte ist von vornherein eine vielschichtige Assemblage aus Verflechtungen, Augenblicken und Wiederkehr.

Die unkonventionelle Datierung des Anthropophagischen Manifests unterstreicht die anachronistische Perspektive: "Ano 374 da Deglutição do Bispo Sardinha" - ein um zwei Jahre versetzter Rückverweis auf den ersten (damals) überlieferten kannibalistischen Akt, die Verspeisung Pedro Fernandes Sardinhas, dem ersten Bischof Brasiliens, der 1556 auf der Reise nach Portugal Schiffbruch erlitt und Indianern zum Opfer fiel. Andrade setzt dieses Ereignis an den Beginn der brasilianischen Zeitrechnung. Doch nicht allein die Zeiten werden durcheinandergewürfelt. Als Ort der Publikation nennt das Manifest Piratininga, was in der Sprache der Tupi das Dorf bezeichnet, auf das die Stadt São Paulo zurückgeht. »Em Piratininga Ano 374 da Deglutição do Bispo Sardinha«: Das Manifest verrückt sowohl temporale als auch lokale und linguistische Komponenten der historiographischen Narration. Die anachronistische Perspektive impliziert eine Ausrahmung des historischen Diskurses, die spielerische Verortung dessen Deterritorialisierung. Diese doppelte Bewegung hat weitreichende Folgen, auch und gerade in der Zusammenschau mit Warburg. Sie lädt dazu ein, die Denkräume kanonischer Kunstgeschichten auszurahmen und verstärkt nach antikolonialen Phänomenen zu fragen.

\section{Vorbilder, Vororte}

Es ist Usus geworden, das Anthropophagische Manifest im Sinne einer bloßen Reversion zu deuten. Demnach wäre die intendierte Bewegung die einer Umkehrung, wie sie wenig später, 1931, auf ironisch-sarkastische Weise im Journal de l'exposition coloniale (Abb.1) in Reaktion auf zeitgenössische Begegnungen zwischen den (Bild-)Kulturen auf die Spitze getrieben werden sollte. $\mathrm{Zu}$ dieser Idee einer umgekehrten Kolonialisierung, für die nunmehr die Ressourcen aus Europa auszubeuten wären, bietet die Lektüre von Kalinca Costa Söderlund eine interessante Alternative. Ausgehend von Kenneth Framptons in Städtebau- und Architekturtheorie eingeführtem Konzept des »kritischen Regionalismus « zeigt die Autorin, inwiefern die Antropofagia-Bewegung eurozentrische Narrationsmuster in der Vermittlung zwischen globalen und lokalen Phänomenen zu unterlaufen versucht: "As a sophisticated Arrière-Garde that not only tackled universal civilisation from without, but also from within, Antropofagia emancipated Brazilian cultural production in relation to that of the centre for it problematized, within a post-colonial reality, cultural and political burdens as heavy as those inherent to the local-global relation. ${ }^{56}$ Damit ist nicht zuletzt auch die Instrumentalisierung der eigenen Geschichtsschreibung angesprochen.

Für Söderlund liegt die Stärke des Programms darin, dass es die Frage der Einverleibung gerade nicht auf der Ebene der Identität verhandelt, sondern als Modus des Austauschs im Sinne einer »fusional alterity«: »the anthropophagous does not find a means of expression through the other, but reproduces the cultural dynamic with which the Tupi cannibal allows the self and the other to merge; to produce culture from the identitarian space in which they converge. $11^{57}$ Die kämpferische Geste des Kannibalen richte sich in erster Linie gegen den internalisierten Kolonialismus der brasilianischen Eliten, nicht gegen die Impulse aus Europa, die vielmehr in ihrer Rolle als Lebenselixier relativiert würden. Demnach speise sich die Bewegung weniger aus dem Bewusstsein der kulturellen Abhängigkeit als aus dem Bestreben, die Mechanismen der Beziehung selbst auszurahmen (statt sie im Modus einer Umkehr, die das Kräfteverhältnis zwar umdreht aber strukturell beibehält, zu bestätigen). Die Einverleibungsmetapher zielt auf eine kritische Geste, die dazu auffordert, Tempo- 
ralitäten und Topographien, die zur Konstruktion von Zentrum und Peripherie geführt haben, umzudenken. Es geht darum, das Nachleben der Bilder ernst zu nehmen, ohne die Vororte (aus) der Geschichte zu verdrängen. In der Antropofagia-Bewegung werden nicht nur Erinnerungstheorie und Geschichtsverständnis verhandelt, sondern auch Kulturbegriffe und Identitätskonzepte. Es gilt, die Gleichrangigkeit zwischen den verschiedenen Elementen zu behaupten, um aus ihrer Begegnung heraus ein Komposit zu entwickeln. Wie bereits die ambivalente Metapher der Anthropophagie suggeriert, werden die Turbulenzen, die damit einhergehen können, nicht verklärt. Nochmals Glissant: »l'éclatement des cultures n'est pas leur éparpillement, ni leur dilution mutuelle. Il est le signe violent de leur partage consenti, non imposé. $\|^{88}$

Die Geste gleicht jenem »dépaysement de la pensée«, von dem François Jullien über den Umweg über China berichtet: kein Ortswechsel,

1 Max Deauville, Exposition de Blancs chez les Noirs, in: Le Journal de l'Exposition coloniale 6, 1931, 3.

2 Viktoria Schmidt-Linsenhoff, Ästhetik der Differenz. Postkoloniale Perspektiven vom 16. bis 21. Jahrhundert, 2 Bde., Marburg 2010, Bd. 1, 21-27.

3 Elizabeth A. Meese und Alice A. Parker (Hg.), The difference within. Feminism and critical theory, Amsterdam/Philadelphia, $\mathrm{Pa} .1989$.

4 Siehe einführend u. a. Carlos Rincón, Avantgarden in Lateinamerika, in: Wolfgang Asholt und Walter Fähnders (Hg.), Der Blick vom Wolkenkratzer. Avantgarde - Avantgardekritik - Avantgardeforschung, Amsterdam 2000, 207-229; Ana Paula Cavalcanti Simioni, Le modernisme brésilien, entre consécration et contestation, in: Perspective. Actualité en histoire de l'art 2, 2013, URL: https://perspective.revues.org/3893 (letzter Zugriff am 10. März 2017).

5 Tarsila do Amaral an ihre Eltern, 19. April 1923, zit. nach Aracy A. Amaral, Tarsila. Sua obra e seu tempo, São Paulo 2003, 101-102, Übersetzung der Autorin. Zu dem Gemälde A Negra u.a. Monica Fauss, »Tupy, or not tupy that is the question«. Drei Gemälde Tarsila do Amarals oder: Primitivismus und Anthropophagismus in der Kunst des brasilianischen Modernismo, in: kritische berichte 25, 1997, Heft 3, 22-42, hier 25-28. Siehe u.a. Tarsila do Amaral sondern Verfremdung zugunsten eines Perspektivwechsels, um sich der eigenen Denkbilder bewusst zu werden - dem »impensé«, »dem NichtGedachten des Denkens «. ${ }^{59}$ Die Karikaturen aus dem Journal de l'exposition coloniale eröffnen keine solchen Kontaktzonen des »in-between «: ${ }^{60}$ Die Bilder stehen im Dienste der Kolonialausstellung und ihrer von Seiten der Gegenausstellung (La vérité sur les colonies) zu Recht kritisierten rassistischen Denkmuster; ${ }^{61}$ sie bewahren und erhärten die tradierten Hierarchien, statt sie aufzubrechen. Sie bilden vertraute Klischees lediglich ab, ohne die Erfahrung des Fremdseins $\mathrm{zu}$ vergegenwärtigen. Die Antropofagia-Bewegung hingegen fordert einen dritten Raum ein, in dem Phänomene von Einverseelung und Einverleibung nicht nur anachronistisch, sondern auch transkulturell gedacht werden können: ein Denkraum deplatzierter Bilder, für den die Frage der Vergegenwärtigung immer auch eine Frage der dekolonialen Verortung wäre.

(Ausst.-Kat. Madrid, Fundación Juan March), hg. von Fundación Juan March, Madrid 2009.

6 Siehe u. a. Kobena Mercer, Black art and the burden of representation, in: Third text. Third World perspectives on contemporary art \& culture 10, 1990, Heft 4, 61-78; Monica Juneja, Global art history and the sburden of representation`, in: Hans Belting, Jakob Birken und Andrea Buddensieg (Hg.), Global studies. Mapping contemporary art and culture, Stuttgart 2011, 274-297.

7 Tarsila do Amaral 1939, zit. nach Amaral 2003 (wie Anm. 5), 249, Übersetzung der Autorin.

8 Oswald de Andrade, Manifesto Antrópofago, in: Revista de Antropofagia 1, 1928, 3 und 7. Im Folgenden zitiert aus der deutschen Übersetzung in: Entre Pindorama (Ausst.-Kat. Stuttgart, Künstlerhaus Stuttgart), hg. von Elke aus dem Moore und Giorgio Ronna, Nürnberg 2005, 26-30. Siehe einführend u. a. Jens Andermann, Antropofagia. Fiktionen der Einverleibung, in: Annette Keck, Inka Kording und Anja Prochaska (Hg.), Verschlungene Grenzen. Anthropophagie in Literatur und Kulturwissenschaften, Tübingen 1999, 19-31; Carlos A. Jáuregui, Antropofagia: consumo cultural, modernidad y utopía, in: ders., Canibalia. Canibalismo, calibanismo, antropofagia cultural y consumo en América Latina, Madrid/Frankfurt a.M. 2008, 393-461. Zur Rolle Tarsilas: Maria 
José Justino, O banquete canibal. A modernidade em Tarsila do Amaral (1886-1973), Curitiba 2002.

9 Michel Foucault, Vorlesung vom 29. Januar 1975, in: ders., Die Anormalen. Vorlesungen am Collège de France (1974-1975), Frankfurt a. M. 2003, 108-142, hier 141, in Bezug auf Kannibalismus und Inzest. Siehe u. a. Joachim Schultz, Kannibalen undsoweiter - Das Fremde und das Komische in der europäischen Avantgarde, in: Ludger Scherer und Rolf Lohse (Hg.), Avantgarde und Komik, Amsterdam/New York 2004, $159-172$.

10 Volker Pantenburg, Faim de Cinéma. Jean-Luc Godard: hungrig, in: Jochen Fritz und Neil Stewart (Hg.), Das schlechte Gewissen der Moderne. Kulturtheorie und Gewaltdarstellung in Literatur und Film nach 1968, Köln 2006, 25-55, hier 26.

11 Rincón 2000 (wie Anm. 4), 212.

12 Siehe Michael Rössner, Spuren der europäischen Avantgarde im smodernistischen Jahrzehnt in Brasilien, in: Harald Wentzlaff-Eggebert (Hg.), Europäische Avantgarde im lateinamerikanischen Kontext, Akten des internationalen Berliner Kolloquiums 1989, Frankfurt a. M. 1991, 31-50, hier 36-39.

13 Haroldo de Campos, Über die anthropophagische Vernunft: Europa im Zeichen des Gefressenwerdens, in: Curt Meyer-Clason (Hg.), Lateinamerikaner über Europa, Frankfurt a. M. 1987, 101-117, hier 102.

14 Thomas Sandführ, Só a Antropofagia nós une. Assimilation und Differenz in der Figur des Anthropophagen, Diss. Universität Düsseldorf 2001, 1314, URL: http://docserv.uni-duesseldorf.de/servlets/ DerivateServlet/Derivate-2302 (letzter Zugriff am 2. März 2017); Jens Badura, Mit dem Anderen anders werden. Kulturtheoretische Sondierungen zum anthropophagischen Manifest, in: Moore und Ronna 2005 (wie Anm. 8), 191-193, hier 193.

15 Hugues Henri, Anthropophagie et hybridation, in: Dominique Berthet (Hg.), Vers une esthétique du métissage?, Paris 2002, 91-107, hier 94. Siehe analog dazu: Suely Rolnik, Anthropophagie zombie, in: Brésil/Europe: repenser le Mouvement Anthropophagique, Kolloquium des Collège international de Philosophie (Paris 2007), Paris 2008, 43-57, URL: http:// www.ciph.org/IMG/pdf/papiers6o.pdf (letzter Zugriff am 10. Februar 2017).

16 Andrade 1928 (wie Anm. 8), 3.

17 Einen guten Eindruck der Lage vermittelt die reiche transatlantische Korrespondenz zwischen Mario de Andrade und Tarsila do Amaral: Aracy Abreu Amaral, Correspondência. Mário de Andrade \& Tarsila do Amaral, São Paulo 1999. Vgl. dazu ihre Veröffentlichungen in: Laura Taddei Brandini (Hg.), Crônicas e outros escritos de Tarsila do Amaral, Campinas 2008.

18 Tarsila do Amaral, Ismos, 1936, in: ebenda, 77-80, hier 79. Entsprechend betont Subirtas: »[P]recisely in their most radical aspects, the philosophical and political views and the conception of culture of Oswald de Andrade and Tarsila do Amaral must be read rather as the very opposite of the futurist cult to the machine and industrialism, and the surrealist scatology of simulacrums and their promise of virtual redemption«. Eduardo Subirtas, From Surrealism to Cannibalism, in: Brasil, 1920-1950. Da antropofagia a Brasília (Ausst.-Kat. São Paulo, MAB-FAAP Museu de Arte Brasileira), hg. von Jorge Schwartz, São Paulo 2002, 529-533, hier 532 .

19 Édouard Glissant, Kreolisierung in der Karibik, in Nord- und Südamerika, in: ders., Kultur und Identität. Ansätze zu einer Poetik der Vielheit, Heidelberg 2013, 7-22, hier 15. Siehe auch ders., Sprachen und Ausdruckweisen, in: ebenda, 23-35, hier 27-28.

20 Badura 2005 (wie Anm. 14), 191.

21 Sandführ 2001 (wie Anm. 14), 4 und 7.

22 Bruno Latour, Nous n'avons jamais été modernes. Essais d'anthropologie symétrique, Paris 1997, 22.

23 Beatriz Sarlo, Una modernidadperiférica. Buenos Aires 1920 y 1930, Buenos Aires 1988. Auch Néstor García Canclini (»hybride Modernität«) und José Joaquín Brunners ("periphere Modernität«) argumentieren zugunsten einer Abgrenzung von Habermas universalistischem Moderne-Projekt: Hermann Herlinghaus und Monika Walter, Lateinamerikanische Peripherie - diesseits und jenseits von Moderne, in: Robert Weimann (Hg.), Ränder der Moderne. Repräsentation und Alterität im (post)kolonialen Diskurs, Frankfurt a.M. 1997, 242-300, hier 252-253; Natascha Ueckmann, Hybriditätskonzepte und Modernekritik in Lateinamerika, in: Wolfgang Klein, Walter Fähnders und Andrea Grewe (Hg.), Dazwischen. Reisen - Metropolen - Avantgarden. Festschrift für Wolfgang Asholt, Bielefeld 2009, 507-529.

24 Zu Recht kritisierte Viktoria Schmidt-Linsenhoff die Perpetuierung tradierter Engpässe im Anschluss an den iconic turn, wenn er wauf eine historische Anthropologie des Bildes abzielt« (Viktoria SchmidtLinsenhoff, Kunst und kulturelle Differenz oder: Warum hat die Kunstgeschichte in Deutschland den spostcolonial turn ausgelassen?, in: Kunst und Politik, Jahrbuch der Guernica-Gesellschaft 4, 2002: Postkolonialismus, 7-17, hier 9). Ihre Forderung nach einem engagierteren Austausch zwischen bildwissenschaftlicher Forschung und Postcolonial Studies ist von ungebrochener Aktualität, bedarf aber inzwischen ihrerseits einer kritischen Revision mit Blick auf verschiedene Entwürfe einer Global Art History. Siehe u.a. Monica Juneja, Kunstgeschichte und kulturelle Differenz. Eine Einleitung, in: kritische berichte 40, 2012, Heft 2, 6-12.

25 Georges Didi-Huberman, Das Nachleben der Bilder. Kunstgeschichte und Phantomzeit nach Aby Warburg, Frankfurt a. M. 2010, 95. 
26 Aby Warburg, Der Bilderatlas Mnemosyne (Gesammelte Schriften, Bd. 2.1), hg. von Martin Warnke, Berlin 2012, 3 .

27 Für eine Differenzierung der Tendenzen: Christian Kravagna, Für eine postkoloniale Kunstgeschichte des Kontakts, in: Texte zur Kunst 91, 2013: Globalismus, $110-131$, besonders 117.

28 Martin Warnke, Vier Stichworte: Ikonologie - Pathosformel - Polarität und Ausgleich - Schlagbilder und Bilderfahrzeuge, in: Werner Hofmann, Georg Syamken und Martin Warnke (Hg.), Die Menschenrechte des Auges. Über Aby Warburg, Frankfurt a. M. 1980, 53-83, hier 75. Siehe Aby Warburg, Manets ,Déjeuner sur l'herbe` [1929/1937], in: Dieter Wuttke (Hg.), Kosmopolis der Wissenschaft. Ernst Robert Curtius und das Warburg Institute. Briefe und andere Dokumente, Baden-Baden 1989, 257-273, hier 262. Vgl. dazu die Editionen des Diktats von 1929 in: Aby Warburg, Bilderreihen und Ausstellungen (Gesammelte Schriften, Bd. 2.2), hg. von Uwe Fleckner und Isabella Woldt, Berlin 2012, 372-375; und in: Martin Treml, Sigrid Weigel und Perdita Ladwig (Hg.), Aby Warburg. Werke in einem Band, Berlin 2010, 647-657.

29 Gerd Blum, Edouard Manet, Le Déjeuner sur l'herbe: Die Erfindung der Moderne aus der Vergangenheit, in: Reingard M. Nischik und Caroline Rosenthal (Hg.), Schwellentexte der Weltiteratur, Konstanz 2001, 201-232.

30 Christoph Zuschlag, Vom Kunstzitat zur Metakunst. Kunst über Kunst im 20. Jahrhundert, in: Wettstreit der Künste. Malerei und Skulptur von Dürer bis Daumier (Ausst.-Kat. München, Haus der Kunst und Köln, Wallraf-Richartz-Museum/Fondation Corboud), hg. von Ekkehard Mai, Wolfratshausen 2002, 170-189, hier 171. Zur Rezeption des Bildes: Jürgen Zänker, Édouard Manets `Déjeuner sur l'herbe in der modernen Kunst und Warenwerbung, in: kritische berichte 8, 1980, Heft 3, 5-31.

31 Warburg 1929/1937 (wie Anm. 28), 262.

32 Warburg 1929 (wie Anm. 28), 655.

33 Ebenda, 268.

34 Ebenda, 262.

35 Ebenda, 268.

36 Warburg zit. nach Cornelia Zumbusch, Wissenschaft in Bildern. Symbol und dialektisches Bild in Aby Warburgs Mnemosyne-Atlas und Walter Benjamins Passagen-Werk, Berlin 2004, 107.

37 Warburg 1929/1937 (wie Anm. 28), 263.

38 Ebenda, 262.

39 Ebenda, Untertitel.

40 Zumbusch 2004 (wie Anm.36), 107. Entsprechend Andersen: "At the same time that he alludes to Giorgione's painting, Manet also disassociates his own picture from it, isolates it from history [...]. It is essential that Manet's references to earlier paintings be recognized as paradoxical." Wayne Andersen, Manet and the Judgment of Paris, in: Art news 2, 1973, $63-69$, hier 65

41 Andrea Pinotti, Archéologie des images et logique rétrospective. Note sur le `Manétisme` de Warburg, in: Images Re-vues, hors-série 4, 2013: Survivance d'Aby Warburg, URL: https://imagesrevues.revues. org/3043 (letzter Zugriff am 12. Dezember 2016).

42 Warburg, 14. April 1928, zit. nach Claudia Wedepohl, Aby Warburg's Theory of Memory, in: Bruniana e Campanelliana 20, 2014, 385- 402, hier 397. Zumbusch unterstreicht die "Betonung der Transformation durch Erinnerung « und leitet daraus aufschlussreiche Verwandtschaften zu Benjamins Begriff des Eingedenkens ab: Zumbusch 2004 (wie Anm. 36), 108.

43 »Jedes wirkliche Kunstwerk ist etwas schlechthin Neues, Nichtdagewesenes. Bei aller Abhängigkeit, Beeinflussung und Stilverwandtschaft bleibt ihm doch ein Charakter der Ausschließlichkeit eigen, der erst das wahre Kunstwerk ausmacht. Darauf beruht ebenso die Unersetzlichkeit des Originals wie die künstlerische Minderwertigkeit selbst einer formal hochqualifizierten Nachbildung." Dagobert Frey, Kunst und Geschichte [1949/1950], in: ders., Bausteine zu einer Philosophie der Kunst, Darmstadt 1976, 1-35, hier 9-10.

44 Pinotti 2013 (wie Anm. 41), 28.

45 Edgar Wind, Einleitung in die Kulturwissenschaftliche Bibliographie zum Nachleben der Antike [1934], in: Wuttke 1989 (wie Anm. 28), 279-294, hier 286.

46 Warburg 1929/1937 (wie Anm. 28), 269. - Eine vergleichende Studie mit Blick auf Jan van der Straets Kupferstich Americen Americus retexit ist als Teil des Forschungsprojektes »Deplatzierte Bilder. Moderne Schauplätze der Kunst« am DFK Paris in Arbeit.

47 Warburg, zit. nach Zumbusch 2004 (wie Anm. 36), 107. Siehe weiterführend vor allem die Beiträge im Rahmen des Projekts Bilderfahrzeuge. Aby Warburg's legacy and the future of iconology, URL: http://iconology.hypotheses.org/ (letzter Zugriff am 7. Februar 2018).

48 Warburg 1929, zit. nach Roland Kany, Mnemosyne als Programm. Geschichte, Erinnerung und die Andacht zum Unbedeutenden im Werk von Usener, Warburg und Benjamin, Tübingen 1987, 185. Der Begriff der Erinnerungsgemeinschaft taucht erstmals 1913 bei Warburg auf. Siehe ebenda, 176. Aus einer anderen Perspektive - über eine Zusammenschau mit Benjamin und Foucault - hat Vinícius Nicastro Honesko eine vergleichende Sicht auf Warburgs Schlangenritual und Andrades Manifest vorgeschlagen, um nach Modellen archäologischer Geschichtsschreibung zu fragen: Vinícius Nicastro Honesko, Da esquizofrenia à antropofagia: leituras da história, in: Confluenze. 
Rivista di studi iberoamericani 1, 2009, Heft 2, 244266.

49 Walter Benjamin, Gesammelte Schriften, Bd. 2.3, hg. von RolfTiedemann und Hermann Schweppenhäuser, Frankfurt a. M. 1991, 1064.

50 Didi-Huberman 2010 (wie Anm. 25), 95.

51 Horst Bredekamp, Bildwissenschaft, in: Ulrich Pfisterer (Hg.), Metzler Lexikon Kunstwissenschaft: Ideen - Methoden - Begriffe, Stuttgart 2004, 56-58, hier 58.

52 Ángel González García, Meditación de los cactus, in: ders., El Resto. Una Historia Invisible del Arte Contemporáneo, Bilbao 2000, 216-231, hier 221. Ich danke Georges Didi-Huberman für den Hinweis auf diesen Text.

53 Didi-Huberman 2010 (wie Anm. 25), 96.

54 Aby Warburg, Schlangenritual. Ein Reisebericht, Berlin 2011, 31-32.

55 Didi-Huberman 2010 (wie Anm. 25), 98.

56 Kalinca Costa Söderlund, Antropofagia: An early Arrière-Garde manifestation in 1920s Brazil, in: RIHA Journal 0132, 2016, URL: http://www.riha-journal. org/articles/2016/0131-0140-special-issue-southernmodernisms/o132-costa-soederlund (letzter Zugriff am 13. Februar 2017).
57 Ebenda, 16.

58 Édouard Glissant, Poétique de la Relation, Poétique III, Paris 1990, 46 - 47 .

59 François Jullien, L'écart et l'entre. Ou comment penser l'altérité (Working Papers Series Fondation maisons des sciences de l'homme, Bd. 3), Paris 2012, URL: https://halshs.archives-ouvertes.fr/halshs-006 77232/document (letzter Zugriff am 23. November 2016); siehe François Jullien, Der Umweg über China. Ein Ortswechsel des Denkens, Berlin 2002, 176-178.

60 Über diese winterstitial spaces" als "spaces where relationships, situations, identities and interactions are shaped through concrete processes of cultural translation«: Doris Bachmann-Medick, Translation - A concept and model for the study of culture, in: Birgit Neumann und Ansgar Nünning (Hg.), Travelling concepts for the study of culture, Berlin/ New York 2012, 23-43, hier 33. Siehe dazu insbesondere: Silviano Santiago, The space in-between. Essays on Latin American culture, Durham/London 2001.

61 Jody Blake, The truth about the colonies, 1931: Art indigène in service of the revolution, in: Oxford Art Journal 1, 2002, 35-58.

Abbildungsnnachweis: 1 Deauville 1931 (wie Anm.1), 3. — 2, 3 Ausst.-Kat. Tarsila do Amaral 2009 (wie Anm. 5), 111 und 30. — 4 XXIV Bienal de São Paulo (Ausst.-Kat., São Paulo, Fundação Bienal São Paulo), 3 Bde., Bd. 1: Núcleo histórico: antropofagia e histórias de canibalismos, São Paulo 1998, A-369. — 5 Manet Manet. Zwei Bilder im Dialog (Ausst.-Kat, London, Courtauld Institute of Art Gallery und München, Neue Pinakothek), hg. von James Cuno und Joachim Kaak, München 2004, 45. 